\title{
FIRST EIGENVALUES OF GEOMETRIC OPERATOR UNDER THE RICCI-BOURGUIGNON FLOW
}

\author{
Shahroud Azami \\ Department of Mathematics, Faculty of Sciences \\ Imam Khomeini International University, Qazvin, Iran \\ azami@sci.ikiu.ac.ir
}

\begin{abstract}
Let $(M, g(t))$ be a compact Riemannian manifold and the metric $g(t)$ evolve by the Ricci-Bourguignon flow. We find the formula variation of the eigenvalues of geometric operator $-\Delta_{\phi}+c R$ under the Ricci-Bourguignon flow, where $\Delta_{\phi}$ is the Witten-Laplacian operator and $R$ is the scalar curvature. In the final section, we show that some quantities dependent to the eigenvalues of the geometric operator are nondecreasing along the Ricci-Bourguignon flow on closed manifolds with nonnegative curvature.
\end{abstract}

Key words and Phrases: Laplace, Ricci-Bourguignon flow.

\begin{abstract}
Abstrak. Misalkan $(M, g(t))$ adalah manifold Riemann kompak dan metrik $g(t)$ berevolusi mengikuti aliran Ricci-Bourguignon. Kita mencari variasi formula nilainilai eigen dari operator geometrik $-\Delta_{\phi}+c R$ di bawah aliran Ricci-Bourguignon, dengan $\Delta_{\phi}$ menyatakan operator Laplace-Witten dan $R$ adalah kurvatur skalar. Di bagian akhir, kita menunjukkan bahwa beberapa besaran yang bergantung pada nilai-nilai eigen dari operator geometrik bersifat tak turun sepanjang aliran RicciBourguignon pada manifold tutup dengan kurvatur taknegatif.
\end{abstract}

Kata kunci: Laplace, aliran Ricci-Bourguignon

\section{INTRODUCTION}

Let $(M, g(t))$ be a closed Riemannian manifold. Studying the eigenvalues of geometric operators is a very powerful tool for the understanding Riemannian manifolds. Recently, there has been a lot of work on the eigenvalue problem under

2000 Mathematics Subject Classification: Primary 58C40; Secondary 53C44.

Received: 31-01-2017, revised: 31-01-2017, accepted: 24-05-2017. 
geometric flow. In [9], Perelman shows that the functional

$$
F=\int_{M}\left(R+|\nabla f|^{2}\right) e^{-f} d \nu
$$

is nondecreasing along the Ricci flow coupled to a backward heat-type equation, where $R$ is the scalar curvature with respect to the metric $g(t)$ and $d \nu$ denote the volume form of the metric $g=g(t)$. The nondecreasing of the functional $F$ implies that the lowest eigenvalue of the operator $-4 \Delta+R$ is nondecreasing along the Ricci flow. As an application, Perelman shown that there are no nontrivial steady or expanding breathers on compact manifolds. Cao [2] extended the geometric operator $-4 \Delta+R$ to the operator $\Delta+\frac{R}{2}$ on closed Riemannian manifolds, and showed that the eigenvalues of the operator $\Delta+\frac{R}{2}$ are nondecreasing along the Ricci flow with nonnegative curvature operator. Then, Li [8] and Cao [3] considered the operator $-\Delta+c R$ and both them proved that the first eigenvalue of the operator $-\Delta+c R$ for $c \geq \frac{1}{4}$ is nondecreasing along the Ricci flow. Zeng and et'al [12] studied the monotonicity of eigenvalues of the operator $-\Delta+c R$ along the Ricci-bourguignon flow. Later Fang and Yang [7] studied the evolution for the first eigenvalue of geometric operator $-\Delta_{\phi}+\frac{R}{2}$ under the Yamabe flow, where $-\Delta_{\phi}$ is the Witten-Laplacian operator, $\phi \in C^{2}(M)$, and constructed some monotonic quantities under the Yamabe flow. Also, Wen and et'al [10] investigated the evolution and monotonicity for eigenvalues of geometric operator $-\Delta_{\phi}+\frac{R}{2}$ under the Ricci flow. For the other recent research in this direction, see $[5,6,11]$

We consider an $n$-dimensional closed Riemannain manifold $M$ with a time dependent Riemannian metric $g(t)$, where $g=g(t)$ is evolving according to the RicciBourguignon flow equation

$$
\frac{\partial}{\partial t} g=-2 R i c+2 \rho R g=-2(R i c-\rho R g), \quad g(0)=g_{0}
$$

where Ric is the Ricci tensor of the manifold, $R$ is scalar curvature and $\rho$ is a real constant. This family of geometric flows contains, as a special case, when $\rho=0$, this flow is the Ricci flow. At the first time the Ricci-Bourguignon introduced by Bourguignon in [1] and then Catino and et 'al in [4] shown that if $\rho<\frac{1}{2(n-1)}$, then the evolution equation (1) has a unique solution for a position time interval on any smooth $n$-dimensional closed Riemannian $M$ with any initial metric $g_{0}$ and shown that some conditions on the curvature are preserved by the Ricci-Bourguignon flow. Motivated by the above works, in this paper we will study the first eigenvalue of the geometric operator whose metric satisfying the Ricci-Bourguignon flow (1).

\section{Preliminaries}

In this section, we will first the definitions for the first eigenvalue of the geometric operator

$$
-\Delta_{\phi}+c R
$$

then we will find the formula for the evolution of the first eigenvalue of the geometric operator (2) under the Ricci-Bourguignon flow on a closed manifold. Let $(M, g(t))$ be a compact Riemannian manifold, and $(M, g(t))$ be a smooth solution to the 
Ricci-Bourguignon flow (1) for $t \in[0, T)$. Let $\nabla$ be the Levi-Civita connection on $(M, g(t))$ and $f: M \rightarrow \mathbb{R}$ be a smooth function on $M$ or $\mathrm{f}$ belong to the Sobolev space $W^{1,2}(M)$. The Laplacian of $f$ defined as

$$
\Delta f=\operatorname{div}(\nabla f)=g^{i j}\left(\partial_{i} \partial_{j} f-\Gamma_{i j}^{k} \partial_{k} f\right) .
$$

Suppose that $d \nu$ the Riemannian volume measure, and $d \mu$ the weight volume measure on $(M, g(t))$; i.e.

$$
d \mu=e^{-\phi(x)} d \nu
$$

where $\phi \in C^{2}(M)$. The Witten-Laplacian is defined by

$$
\Delta_{\phi}=\Delta-\nabla \phi . \nabla
$$

which is a symmetric operator on $L^{2}(M, \mu)$ and satisfies the following integration by part formula:

$$
\int_{M}<\nabla u, \nabla v>d \mu=-\int_{M} v \Delta_{\phi} u d \mu=-\int_{M} u \Delta_{\phi} v d \mu \quad \forall u, v \in C^{\infty}(M),
$$

The Witten-Laplacian is generalize of Laplacian operator, for example, when $\phi$ is a constant function, the Witten-Laplacian operator is just the Laplace-Belterami operator. In this paper we consider a generalize of the Witten-Laplacian operator as $-\Delta_{\phi}+c R$ where $R$ is the scalar curvature. We say that $\lambda(t)$ is an eigenvalue of the operator $-\Delta_{\phi}+c R$ at time $t \in[0, T)$, and $f(x, t)$ the corresponding eigenfunction, whenever

$$
-\Delta_{\phi} f(x, t)+c R f(x, t)=\lambda(t) f(x, t) .
$$

Normalized eigenfuctions are defined as follow:

$$
\int_{M} f^{2} d \mu=1
$$

and assume that $f(x, t)$ is a $C^{1}$-family of smooth function on $M$.

Multiplying with $f$ on both sides (6) and then by integration we get

$$
\lambda(t)=\int_{M}\left(-f \Delta_{\phi} f+c R f^{2}\right) d \mu
$$

where defines the evolution of the first eigenvalue of the geometric operator (2) under the variation of $g(t)$ where the eigenfunction associated to $\lambda(t)$ is normalized. In [10] Wen and et 'al shown that the following lemma.

Lemma 2.1. ([10]) Suppose that $\lambda(t)$ is an eigenvalue of the operator $-\Delta_{\phi}+c R, f$ is the eigenfunction of $\lambda(t)$ at the time $t$, and the metric $g(t)$ evolves by $\frac{\partial}{\partial t} g_{i j}=v_{i j}$, where $v_{i j}$ is a symmetric two-tensor. Then we have

$$
\frac{d}{d t} \lambda(t)=\int_{M}\left(v_{i j} f_{i j}-v_{i j} \phi_{i} f_{j}+c \frac{\partial R}{\partial t} f\right) d \mu+\int_{M}\left(v_{i j, i}-\frac{1}{2} V_{i}\right) f_{j} f d \mu
$$

where $V=\operatorname{tr}(v), f_{i j}=\nabla_{i} \nabla_{j} f, f_{i}=\nabla_{i} f, \phi_{i}=\nabla_{i} \phi$.

Now, we find the evolution formula of eigenvalue $\lambda(t)$ under the Ricci-Bourguignon flow (1). 
Theorem 2.2. Let $g(t), t \in[0, T)$, be a solution of the Ricci-Bourguignon flow (1) on an $n$-dimensional closed manifold $M$. Assume that $\lambda(t)$ is the lowest eigenvalue of $-\Delta_{\phi}+c R$ and $f=f(x, t)$ satisfies in (6) with (7). Then under the RicciBourguignon flow, we have

$$
\begin{aligned}
\frac{d}{d t} \lambda(t)= & 2 A \int_{M} R\left|\frac{1}{2} \nabla f-f \nabla \phi\right|^{2} d \mu-A \int_{M} R f^{2} \Delta \phi d \mu \\
& +\left[\frac{3}{2} A-1+(n-2) \rho\right] \int_{M} R|\nabla f|^{2} d \mu-\lambda(2 A-1+n \rho) \int_{M} R f^{2} d \mu \\
& +2 \int_{M} R_{i j} f_{i} f_{j} d \mu+2 c \int_{M}|R i c|^{2} f^{2} d \mu+c(2 A-1+(n-2) \rho) \int_{M} R^{2} f^{2} d \mu
\end{aligned}
$$

where $A=c(1-2(n-1) \rho)$.

Proof. In [4], G. Catino and et'al shown that the evolution of scalar curvature under the Ricci-Bourguignon flow is

$$
\frac{\partial R}{\partial t}=(1-2(n-1) \rho) \Delta R+2|R i c|^{2}-2 \rho R^{2} .
$$

Substiuting $v_{i j}=-2 R_{i j}+2 \rho R g_{i j}$ and (11) into the equality (9) we get

$$
\begin{aligned}
\frac{d}{d t} \lambda(t)= & \int_{M}\left[-2 R_{i j} f_{i j}+2 \rho R \Delta f+2 R_{i j} \phi_{i} f_{j}-2 \rho R \nabla \phi . \nabla f\right] f d \mu \\
& -(n-2) \rho \int_{M} \nabla_{i} R f_{i} f d \mu \\
& +c \int_{M}\left[(1-2(n-1) \rho) \Delta R f^{2}+2|R i c|^{2} f^{2}-2 \rho R^{2} f^{2}\right] d \mu .
\end{aligned}
$$

Integration by parts results that

$$
\begin{aligned}
\int_{M} f^{2} \Delta R d \mu= & 2 \int_{M} R|\nabla f|^{2} d \mu+2 \int_{M} R f \Delta_{\phi} f d \mu-2 \int_{M} R f \nabla \phi . \nabla f d \mu \\
& -\int_{M} R f^{2} \Delta \phi d \mu+\int_{M} R f^{2}|\nabla \phi|^{2} d \mu
\end{aligned}
$$

and

$$
\int_{M} \nabla_{i} R f_{i} f d \mu=-\int_{M} R f \Delta_{\phi} f d \mu-\int_{M} R|\nabla f|^{2} d \mu .
$$


Also, using integration by parts and $\frac{1}{2} \nabla R=$ div Ric we have

$$
\begin{aligned}
-\int_{M} R_{i j} f_{i j} f d \mu & =\int_{M}\left(R_{i j} f e^{-\phi}\right)_{j} f_{i} d \nu \\
& =\int_{M} \operatorname{div}(R i c) f e^{-\phi} f_{i} d \nu+\int_{M} R_{i j} f_{j} e^{-\phi} f_{i} d \nu-\int_{M} R_{i j} f \phi_{j} f_{i} e^{-\phi} d \nu \\
& =\frac{1}{2} \int_{M} \nabla_{i} R f f_{i} e^{-\phi} d \nu+\int_{M} R_{i j} f_{j} f_{i} d \mu-\int_{M} R_{i j} f \phi_{j} f_{i} d \mu \\
& =-\frac{1}{2} \int_{M} R\left(f f_{i} e^{-\phi}\right)_{i} d \nu+\int_{M} R_{i j} f_{j} f_{i} d \mu-\int_{M} R_{i j} f \phi_{j} f_{i} d \mu \\
& =-\frac{1}{2} \int_{M} R|\nabla f|^{2} d \mu-\frac{1}{2} \int_{M} R f \Delta f d \mu+\frac{1}{2} \int_{M} R f f_{i} \phi_{i} d \mu \\
& +\int_{M} R_{i j} f_{j} f_{i} d \mu-\int_{M} R_{i j} f \phi_{j} f_{i} d \mu \\
= & -\frac{1}{2} \int_{M} R|\nabla f|^{2} d \mu-\frac{1}{2} \int_{M} R f \Delta_{\phi} f d \mu+\int_{M} R_{i j} f_{j} f_{i} d \mu-\int_{M} R_{i j} f \phi_{j} f_{i} d \mu .
\end{aligned}
$$

Inserting (13), (14) and (15) in (12), yields

$$
\begin{aligned}
\frac{d}{d t} \lambda(t)= & -\int_{M} R|\nabla f|^{2} d \mu-\int_{M} R f \Delta_{\phi} f d \mu+2 \int_{M} R_{i j} f_{i} f_{j} d \mu-2 \int_{M} R_{i j} f_{i} \phi_{j} f d \mu \\
& +2 \rho \int_{M} R f \Delta f d \mu+2 \int_{M} R_{i j} f_{j} \phi_{i} f d \mu-2 \rho \int_{M} R f \nabla \phi . \nabla f d \mu \\
& +(n-2) \rho \int_{M} R f \Delta_{\phi} f d \mu+(n-2) \rho \int_{M} R|\nabla f|^{2} d \mu \\
& +2 c(1-2(n-1) \rho) \int_{M} R|\nabla f|^{2} d \mu+2 c(1-2(n-1) \rho) \int_{M} R f \Delta_{\phi} f d \mu \\
& -2 c(1-2(n-1) \rho) \int_{M} R f \nabla \phi . \nabla f d \mu-c(1-2(n-1) \rho) \int_{M} R f^{2} \Delta \phi d \mu \\
& +2 c(1-2(n-1) \rho) \int_{M} R f^{2}|\nabla \phi|^{2} d \mu+2 c \int_{M}|R i c|^{2} f^{2} d \mu-2 c \rho \int_{M} R^{2} f^{2} d \mu,
\end{aligned}
$$


therefore

$$
\begin{aligned}
\frac{d}{d t} \lambda(t)= & {[2 A-1+(n-2) \rho] \int_{M} R|\nabla f|^{2} d \mu+[2 A-1+n \rho] \int_{M} R f \Delta_{\phi} f d \mu } \\
& +2 \int_{M} R_{i j} f_{i} f_{j} d \mu+2 c \int_{M}|R i c|^{2} f^{2} d \mu-2 c \rho \int_{M} R^{2} f^{2} d \mu \\
& -2 A \int_{M} R f \nabla \phi . \nabla f d \mu-A \int_{M} R f^{2} \Delta \phi d \mu+2 A \int_{M} R f^{2}|\nabla \phi|^{2} d \mu \\
= & 2 A \int_{M} R\left|\frac{1}{2} \nabla f-f \nabla \phi\right|^{2} d \mu-A \int_{M} R f^{2} \Delta \phi d \mu \\
& +\left[\frac{3}{2} A-1+(n-2) \rho\right] \int_{M} R|\nabla f|^{2} d \mu \\
& +(2 A-1+n \rho) \int_{M} R\left(-\lambda f^{2}+c R f^{2}\right) d \mu \\
& +2 \int_{M} R_{i j} f_{i} f_{j} d \mu+2 c \int_{M}|R i c|^{2} f^{2} d \mu-2 c \rho \int_{M} R^{2} f^{2} d \mu .
\end{aligned}
$$

Here in the last equality we have used (6).

In theorem (2.2) if $\phi$ is a constant function, we can get the evolution for the first eigenvalue of the geometric operator $-\Delta+c R$ under the Ricci-Bourguignon flow (1), which studied in [12].

Remark: In theorem (2.2), we assume that $\phi$ does not dependent on the time $t$. If $\phi$ is depend to $t$ then the eigenvalue $\lambda(t)$ introduced in (6) and (7) satisfies

$$
\begin{aligned}
\frac{d}{d t} \lambda(t)= & 2 A \int_{M} R\left|\frac{1}{2} \nabla f-f \nabla \phi\right|^{2} d \mu-A \int_{M} R f^{2} \Delta \phi d \mu+\int_{M} f_{i}\left(\phi_{t}\right)_{i} f d \mu \\
& +\left[\frac{3}{2} A-1+(n-2) \rho\right] \int_{M} R|\nabla f|^{2} d \mu-\lambda(2 A-1+n \rho) \int_{M} R f^{2} d \mu \\
& +2 \int_{M} R_{i j} f_{i} f_{j} d \mu+2 c \int_{M}|R i c|^{2} f^{2} d \mu \\
& +c(2 A-1+(n-2) \rho) \int_{M} R^{2} f^{2} d \mu
\end{aligned}
$$

where $A=c(1-2(n-1) \rho)$. Because the evolution equation of eigenvalues will have an additional term $\int_{M} f_{i}\left(\phi_{t}\right)_{i} f d \mu$.

In the following we show that some quantity dependent on the eigenvalue of geometric operator (6) are monotonic along the Ricci-Bourguignon flow. Not that the scalar curvature under the Ricci-Bourguignon flow evolves by

$$
\frac{\partial R}{\partial t}=(1-2(n-1) \rho) \Delta R+2|R i c|^{2}-2 \rho R^{2},
$$

by $|R i c|^{2} \leq R^{2}$ we have

$$
\frac{\partial R}{\partial t} \leq(1-2(n-1) \rho) \Delta R+2(1-\rho) R^{2}
$$


Let $\sigma(t)$ be the solution to the ODE $y^{\prime}=2(1-\rho) y^{2}$ with initial value $\alpha=\max _{x \in M} R(0)$. By the maximum principle, we have

$$
R(t) \leq \sigma(t)=\left(-2(1-\rho) t+\frac{1}{\alpha}\right)^{-1}
$$

on $\left[0, T^{\prime}\right)$, where $T^{\prime}=\min T, \frac{1}{2(1-\rho) \alpha}$. Also, the inequality $|R i c|^{2} \geq \frac{R^{2}}{n}$ result that

$$
\frac{\partial R}{\partial t} \geq(1-2(n-1) \rho) \Delta R+2\left(\frac{1}{n}-\rho\right) R^{2} .
$$

we assume that $\gamma(t)$ be the solution to the ODE $y^{\prime}=2\left(\frac{1}{n}-\rho\right) y^{2}$ with initial value $\beta=\min _{x \in M} R(0)$. Then the maximum principle implies that

$$
R(t) \geq \gamma(t)=\frac{n \beta}{n-2(1-n \rho) \beta t} \text { on }[0, T) .
$$

Theorem 2.3. Let $(M, g(t))$ be a solution of the Ricci-Bourguignon flow (1) for $t \in[0, T]$ on a closed $n$-dimensional manifold $M$ and $\rho<\frac{1}{2(n-1)}$ with nonnegative scalar curvature. Let the Ricci curvature operator be a nonnegative along the RicciBourguignon flow and scalar curvature satisfies

$$
R \geq \frac{1-2(n-1) \rho}{2 A-1+(n-2) \rho} \Delta \phi, \quad \text { in } M \times[0, T] .
$$

If $\lambda(t)$ is the first eigenvalue of (2) then for $c \geq \frac{2(1-(n-2) \rho)}{3(1-2(n-1) \rho)}$, the quantity

$$
e^{\int_{0}^{t}[-(2 A+n \rho) \gamma(\tau)+\sigma(\tau)] d \tau} \lambda(t)
$$

is nondecreasing under the Ricci-Bourguignon flow on $\left[0, T^{\prime}\right)$ where $A=c(1-2(n-$ 1) $\rho)$ and $\sigma(t)$ and $\gamma(t)$ introduced in (18) and (19), respectively.

Proof. According to hypothesis of the theorem the Ricci curvature operator is nonnegative along the Ricci-Bourguignon flow and on the other hand in [4], shown that the nonnegative of the scalar curvature is preserved along the Ricci-Bourguignon flow. Therefore (10) and (20) imply that for $\rho<\frac{1}{2(n-1)}$ and $c \geq \frac{2(1-(n-2) \rho)}{3(1-2(n-1) \rho)}$ we get

$$
\frac{d}{d t} \lambda(t) \geq \lambda(2 A-1+n \rho) \int_{M} R f^{2} d \mu \geq \lambda[(2 A+n \rho) \gamma(t)-\sigma(t)] .
$$

in last inequality we used $\int_{M} f^{2} d \mu=1$. Hence the theorem follows from the last inequality.

Theorem 2.4. Let $g(t), t \in[0, T)$ be a solution to the Ricci-Bourguignon flow (1) on a closed Riemannian manifold $M^{n}$ with nonnegative scalar curvature and the scalar curvature satisfies

$$
R \geq \frac{2(n-1)}{4 c(n-1)-n+2} \Delta \phi, \quad \text { in } M \times[0, T) .
$$


Suppose that $n \geq 3$ and the Ricci curvature satisfies

$$
\left|R i c-\frac{1}{4 c-1} \nabla \nabla \phi\right|^{2} \geq \frac{4 c}{(4 c-1)^{2}}|\nabla \nabla \phi|^{2}, \quad \text { in } M \times[0, T)
$$

where $c>\frac{n-2}{2(n-1)}$ and $\phi \in C^{\infty}(M)$ satisfies the heat equation

$$
\frac{\partial \phi}{\partial t}=\Delta \phi
$$

Then for $\rho \leq 0$ the quantity $e^{-\int_{0}^{t}(-\rho n \gamma(\tau)+4 \rho c(n-1) \sigma(\tau)) d \tau} \lambda(t)$ is nondecreasing along the Ricci-Bourguignon flow, where $\sigma(t)$ and $\gamma(t)$ introduced in (18) and (19), respectively.

Proof. From (12) and (17), we have

$$
\begin{aligned}
\frac{d}{d t} \lambda(t)= & -2 \rho \int_{M}\left[-R f \Delta f+R f \phi_{i} f_{i}+\frac{n-2}{2} \nabla_{i} R f_{i} f\right. \\
& \left.+c(n-1) \Delta R f^{2}+c R^{2} f^{2}\right] d \mu \\
& +\int_{M}\left[-2 R_{i j} f_{i j} f+2 R_{i j} \phi_{i} f_{j} f+c \Delta R f^{2}+2 c|R i c|^{2} f^{2}+f_{i}\left(\phi_{t}\right)_{i} f\right] d \mu
\end{aligned}
$$

we set

$$
I=\int_{M}\left[-R f \Delta f+R f \phi_{i} f_{i}+\frac{n-2}{2} \nabla_{i} R f_{i} f+c(n-1) \Delta R f^{2}+c R^{2} f^{2}\right] d \mu
$$

and

$$
I I=\int_{M}\left[-2 R_{i j} f_{i j} f+2 R_{i j} \phi_{i} f_{j} f+c \Delta R f^{2}+2 c|R i c|^{2} f^{2}+f_{i}\left(\phi_{t}\right)_{i} f\right] d \mu .
$$

Notice that, using (13) and (14) we can rewrite $I$ as follow:

$$
\begin{aligned}
I= & c(n-1) \int_{M} R|\nabla f-f \nabla \phi|^{2} d \mu+\left[-\frac{n}{2} c+2 c^{2}(n-1)+c\right] \int_{M} R^{2} f^{2} d \mu \\
& +\left[-\left(\frac{n}{2}-1\right)+c(n-1)\right] \int_{M} R|\nabla f|^{2} d \mu-\left[-\frac{n}{2}+2 c(n-1)\right] \lambda \int_{M} R f^{2} d \mu \\
& -c(n-1) \int_{M} R f^{2} \Delta \phi d \mu,
\end{aligned}
$$

on the other hand, in [10], has been shown that

$$
\begin{aligned}
I I= & \frac{1}{2} \int_{M}\left|R_{i j}+\psi_{i j}\right|^{2} e^{-\psi} d \mu+\frac{4 c-1}{2} \int_{M}|R i c|^{2} e^{-\psi} d \mu \\
& +\int_{M}\left(\psi_{i j} \phi_{i j}+\frac{1}{2} \psi_{i}(\Delta \phi)_{i}\right) e^{-\psi} d \mu-\frac{1}{2} \int_{M} \psi_{i}\left(\phi_{t}\right)_{i} e^{-\psi} d \mu
\end{aligned}
$$

where $f^{2}=e^{-\psi}$ for some smooth function $\psi$. Therefore if $\frac{\partial \phi}{\partial t}=\Delta \phi$ then we have

$$
-\frac{1}{2} \int_{M} \psi_{i}\left(\phi_{t}\right)_{i} e^{-\psi} d \mu=-\frac{1}{2} \int_{M} \psi_{i}(\Delta \phi)_{i} e^{-\psi} d \mu .
$$


Combining (25), (26), (27) and (28) we arrive at

$$
\begin{aligned}
\frac{d}{d t} \lambda(t)= & \frac{1}{2} \int_{M}\left|R_{i j}+\psi_{i j}\right|^{2} e^{-\psi} d \mu+\frac{4 c-1}{2} \int_{M}|R i c|^{2} e^{-\psi} d \mu+\int_{M} \psi_{i j} \phi_{i j} e^{-\psi} d \mu \\
& -2 \rho c(n-1) \int_{M} R\left|\frac{1}{2} \nabla \psi-\nabla \phi\right|^{2} e^{-\psi} d \mu \\
& -2 \rho\left[-\frac{n}{2} c+2 c^{2}(n-1)+c\right] \int_{M} R^{2} e^{-\psi} d \mu \\
& +\frac{\rho}{2}\left[\left(\frac{n}{2}-1\right)-c(n-1)\right] \int_{M} R|\nabla \psi|^{2} e^{-\psi} d \mu \\
& +2 \rho\left[-\frac{n}{2}+2 c(n-1)\right] \lambda \int_{M} R e^{-\psi} d \mu+2 \rho c(n-1) \int_{M} R e^{-\psi} \Delta \phi d \mu \\
= & \frac{1}{2} \int_{M}\left|R_{i j}+\psi_{i j}+\phi_{i j}\right|^{2} e^{-\psi} d \mu \\
& +\frac{4 c-1}{2} \int_{M}\left(\left|R_{i j}-\frac{1}{4 c-1} \phi_{i j}\right|^{2}-\frac{4 c}{(4 c-1)^{2}}\left|\phi_{i j}\right|^{2}\right) e^{-\psi} d \mu \\
& -2 \rho c(n-1) \int_{M} R\left|\frac{1}{2} \nabla \psi-\nabla \phi\right|^{2} e^{-\psi} d \mu \\
& -2 \rho\left[-\frac{n}{2} c+2 c^{2}(n-1)+c\right] \int_{M} R^{2} e^{-\psi} d \mu \\
& +\frac{\rho}{2}\left[\left(\frac{n}{2}-1\right)-c(n-1)\right] \int_{M} R|\nabla \psi|^{2} e^{-\psi} d \mu \\
& +2 \rho\left[-\frac{n}{2}+2 c(n-1)\right] \lambda \int_{M} R e^{-\psi} d \mu+2 \rho c(n-1) \int_{M} R e^{-\psi} \Delta \phi d \mu .
\end{aligned}
$$

The nonnegative scalar curvature is preserved along the Ricci-Bourguignon flow, then (22) and (23) for $\rho \leq 0$ and $c>\frac{n-2}{2(n-1)}$ imply that

$$
\frac{d}{d t} \lambda(t) \geq 2 \rho\left[-\frac{n}{2}+2 c(n-1)\right] \lambda \int_{M} R e^{-\psi} d \mu \geq[-\rho n \gamma(t)+4 \rho c(n-1) \sigma(t)] \lambda
$$

the last inequality complete the proof of theorem.

If we assume that $\phi$ is constant function and $\rho=0$ then (29) implies that for $c \geq \frac{1}{4}$ the eigenvalues is strictly increasing under the Ricci-Bourguignon flow, where this result funded by Cao in [3].

\section{REFERENCES}

[1] Bourguignon, J.P., "Ricci curvature and Einstein metrics, Global differential geometry and global analysis" (Berlin,1979) Lecture nots in Math. vol. 838, Springer, Berlin, 1981, 42-63.

[2] Cao, X.D., "Eigenvalues of $\left(-\Delta+\frac{R}{2}\right)$ on manifolds with nonnegative curvature operator", Math. Ann. 337:2(2007), 435-441. 
[3] Cao, X.D., "First eigenvalues of geometric operators under the Ricci flow",Proc. Amer. Math. Soc., 136(2008), 4075-4078

[4] Catino, G., Cremaschi, L., Djadli, Z., Mantegazza, C., and Mazzieri, L., "The RicciBourguignon flow", Pacific J. Math., (2015).

[5] Cerbo, L.F.D., "Eigenvalues of the Laplacian under the Ricci flow", Rendiconti di Mathematica, Serie VII, 27(2007), 183-195.

[6] Cheng, Q.-M., and Yang, H.C., "Estimates on eigenvalues of Laplacian",Math. Ann., 331(2005), 445-460

[7] Fang, S., and Yang, F., "First eigenvalues of geometric operators under the Yamabe flow", Bull. Korean Math. Soc., 53(2016), 1113-1122.

[8] Li, J.F., "Eigenvalues and energy functionals with monotonicity formula under Ricci flow", Math. Ann., (2007), 338, 927-946.

[9] Perelman, G., "The entropy formula for the Ricci flow and its geometric applications", (2002), ArXiv: 0211159.

[10] Wen, F.S., Feng, X.H., and Peng, Z., "Evolution and monotonicity of eigenvalues under the Ricci flow",Sci. China Math., 58(2015),no. 8, 1737-1744.

[11] Wu, J.Y., "First eigenvalue monotonicity for the $p$-Laplace operator under the Ricci flow", Acta mathematica Sinica, English senes, 27:8(2011), 1591-1598.

[12] Zeng, F., He, Q., and Chen, B., "Monotonicity of eigenvalues of geometric operators along the Ricci-Bourguignon flow", Arxiv, 152.08158v1. 